政

\title{
Unusual stroke mimic
}

\begin{abstract}
We report a case of a 69 year old right handed female, presents to Emergency Room (ER) with sudden onset right hemiparesis, within 90 minutes. She also had nagging neck pain since the start of this weakness. Her examination showed dense right hemiplegia with minimal involvement of face, her initial CT brain was unremarkable. As she was within therapeutic window, was given IV Fibrinolytic therapy. Within three hours, she developed left hemiparesis while her neck pain worsened. Repeat CT brain was unremarkable. Neurologic examination at this stage showed she had quadraparesis with left hemi-sensory loss and new development of right sided horner's syndrome. MRI brain was unremarkable, MRI cervical spine showed spindle shaped extradural mass, likely tumoral hematoma, extending from C2-C6 compressing spinal cord with edema. Neurosurgical evaluation prompted urgent cervical decompression with improvement in her motor function. This case highlights usual presentation with a rarest reason mimicking stroke.
\end{abstract}

Volume 7 Issue 7 - 2017

\author{
Khurram A Siddiqui,' Renganathan \\ Radhakrishanan, ' Ali R Dehdashtian, ${ }^{2}$ Irfan \\ Elahi, ${ }^{3}$ Shahzad Maqbool ${ }^{3}$ \\ Department of Neurology, Al Ain Hospital, UAE \\ ${ }^{2}$ Department of Radiology, Al Ain Hospital, UAE \\ ${ }^{3}$ Department of Neurosurgery, Al Ain Hospital, UAE
}

Correspondence: Khurram A Siddiqui, Consultant Neurologist and Epileptologist, Adjunct Associate Professor of Neurology, Department of Neurology, Medical Institute, Al Ain Hospital, SEHA Group of hospitals, AI Ain, UAE, Email ksiddiqui@seha.ae

Received: September 22, 2017 | Published: November 21 , 2017

\section{Introduction}

This interesting case is important to be written up as the presentation was usual, stroke- right hemiplegia and was presented as such and was within therapeutic window and was given IV Fibrinolytic therapy as per hospital stroke guideline and within few hours she developed left sided weakness and continued to have neck pain which was thought initially on presentation to be related to possible cervical dissection and her development of new symptoms and signs lead to investigations of the cervical spine that yielded the diagnosis of a extradural spinal mass. This case highlights proper neurologic examination and reexamination of the patient can lead to diagnosis and management.

\section{Case report}

A 69 year old right handed female presents to ER with sudden onset right sided weakness. She had past medical history of diabetes mellitus, hypertension, hyperlipidemia and obesity. She developed right upper and lower extremity weakness which progressed by the time she presented to ER and she was unable to move her right side. She presented within 90 minutes and was initially examined by ER Physician within a minute of her presentation, stroke code (as per hospital's ischemic stroke guideline) was activated which lead to urgent CT Brain within 15 minutes and was examined by Neurologist within 30 minutes of her presentation. Quick history and collateral history, excluding the relative and absolute contraindications, neurologist noted she had right sided neck pain and had right sided hemiplegia. Neurologic examination at this stage showed she had flattened right naso-labial fold, right upper extremity power was $0 / 5$ and right lower extremity was $0 / 5$, without any sensory loss. She had absent reflexes all over and reduced tone and planters was extensor on the right and was flexor on the left side. Her NIHSS (National Institute of Health Stroke Scale) was 9. As initial CT brain was unremarkable, her family was consented for Fibrinolytic therapy with IV rtPA (recombinant tissue Plasminogen Activator) and was dispensed at a dose of $0.9 \mathrm{mg} / \mathrm{kg}$ within 45 minutes of her presentation. While in the transit from ER to Stroke Unit that is within 3 hours post her IV rtPA, she started to complain that she can't feel her left leg and arm and has developed left sided weakness. This prompted a repeat CT brain which was unremarkable for any signs of hemorrhage and or ischemia. Re-examination at this stage showed she had quadraparesis, with dense right hemiplegia of power $0 / 5$ in the right upper and lower extremity and also left hemiparesis with power $1 / 5$ in the left lower extremity and $2 / 5$ in the left upper extremity with reduced tone all over and absent reflexes. Her Babinski's response was extensor on the right side and was unequivocal on the left side. She also had left hemi-sensory loss to pinprick and temperature sense up to her neck sparing the face along with right horner's (ptosis, smaller pupil and enopthalmos) with preserved extra ocular movements. Her neck pain had worsened and has been requiring opioid analgesics, with new development of her symptoms and signs this lead to further neuroimaging in the form of MRI Brain Stroke Protocol which was unremarkable and MRI Cervical spine which showed spindle-shaped extradural lesion extending from $\mathrm{C} 2$ to $\mathrm{C} 6$ exerting significant mass effect on the thecal sac and related spinal cord with subsequent cord edema. The lesion predominantly is of low signal in T2 and intermediate signal in T1 displaying tiny foci of enhancement after administration of contrast, suggesting underlying tumor with a surrounding hematoma, Figure 1. Emergent neurosurgical evaluation prompted urgent cervical decompression in the form of right hemilaminectomy from $\mathrm{C} 2$ to $\mathrm{C} 5$ which revealed extra-dural tumor with clotted hematoma which was sub- acute, compressing the spinal cord this was excised, Figure 2, and with resultant improvement in her left sided motor function, she remained weak on the right side and has been treated with IV dexamethasone and with aggressive physiotherapy. This case highlights usual presentation with a rarest reason mimicking stroke.

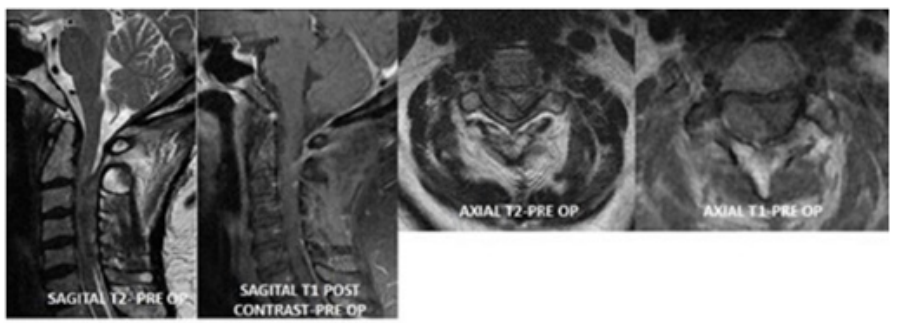

Figure I Pre-Operative; MRI of Cervical Spine with and without contrast showed spindle-shaped extradural lesion extending from $\mathrm{C} 2$ to $\mathrm{C} 6$ exerting significant mass effect on the thecal sac and related spinal cord with subsequent cord edema. The lesion predominantly is of low signal in T2 and intermediate signal in TI displaying tiny foci of enhancement after administration of contrast.

\section{Discussion}

Treatment of acute stroke presenting in a therapeutic window has been widely accepted and practiced and almost all parts of the world. 
If recognized acute stroke and not given IV Fibrinolytic therapy within time frame $(<4.5$ hours $)$ can have devastating outcomes ranging from severe disability to death for some patients and has legal implications in some countries. Most common stroke mimics are; seizure followed by todd's paresis, migraine complicated by hemiplegic migraine, Psychogenic hemiparesis, hypertensive encephalopathy and hypoglycemia. Spinal cord tumor/hematoma can rarely mimic a stroke as the presentation is usually of one of the spinal cord syndromes and can range from anterior cord syndrome, posterior cord syndrome, central cord syndrome and rarely hemi-cord syndrome. We think our patient presented as partial hemi-cord syndrome which made us think that this is an acute ischemic stroke and with a normal CT brain was given IV Fibrinolytic therapy. Although the clue was she had neck pain but thinking in the context of stroke it could be secondary to spontaneous cervical dissection.

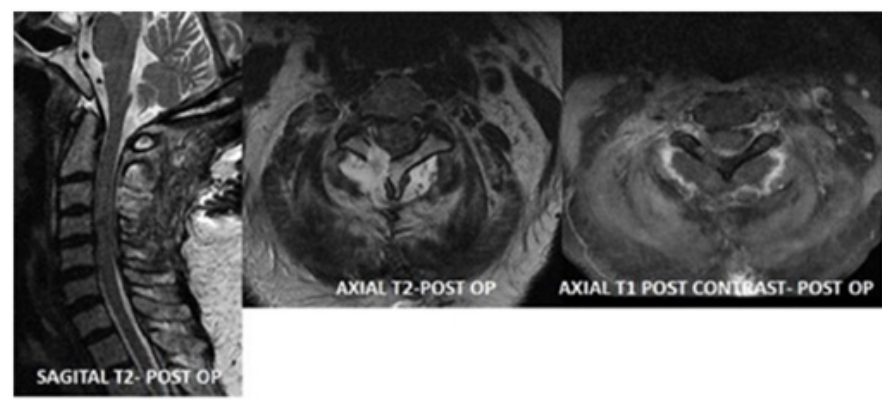

Figure 2 Post-Operative; MRI of Cervical Spine with and without contrast showed status-post right hemi-laminectomy with successful evacuation of intra-spinal extradural mass lesion. Fluid signal seen in right postero-lateral aspect of spine likely due to postoperative changes.

This has been reported in literature as SSEH (spontaneous spinal epidural hematoma) as Hiroaka et al., ${ }^{1}$ have reported two cases who presented as acute stroke and one of their patients presenting as hemiparesis with neck pain similar to our case and other patient with hemiparesis with Lhermitt's sign, both of these patients were not thrombolysed unlike our case as they had MRI brain scan to exclude stroke and were found to have spontaneous spinal epidural hematoma, on cervical neuroimaging. There were multiple case reports from different parts of the world mostly reported in spine and neurosurgical literature,,$^{2-5}$ regarding spontaneous epidural hematoma. ${ }^{6}$ Our case was different in the sense patient that there was a hematoma around the tumor as seen by the operating neurosurgeon and was removed enblock, further investigations regarding possible metastasis are underway. It's possible that patient worsening seen after admission was seen after thrombolytic therapy could be because of bleeding in the tumor but on gross examination by neurosurgeon he found clotted blood which was sub-acute. Our case highlights that neck pain should be taken in consideration in patients with acute stroke and cervical neuroimaging should be offered to patients with acute hemiparesis, leading to appropriate diagnosis and management and avoidance of thrombolytic therapy. Fortunately this did not cause any detrimental effects in our patients but could be devastating.

\section{Conflicts of interest}

None.

\section{Acknowledgements}

None.

\section{Funding}

None.

\section{References}

1. Caplan LR. et al. Top of the basilar syndrome. Neurology. 1980;30(1):72-79.

2. Matsumoto H, Miki T, Miyaji Y, et al. Spontaneous spinal epidural hematoma with hemiparesis mimicking acute cerebral infarction: Two case reports. J Spinal Cord Med. 2012;35(4):262-266.

3. Tanaka A, Nagakane Y, Hosomi A, et al. A case of acute spontaneous spinal epidural hematoma with sudden onset of hemiplegia under consideration for intravenous alteplase. Jpn J Stroke. 2008;30:60-63

4. Usami K, Tokumoto K, Ino $\mathrm{T}$, et al. Cervical epidural hematoma mimicking stroke: case report. Jpn J Stroke. 2008;30:516-520.

5. Wang $\mathrm{CC}$, Chang $\mathrm{CH}$, Lin $\mathrm{HJ}$,et al. Misdiagnosis of spontaneous cervical epidural haemorrhage. Eur Spine J. 2009;18Suppl 2:210-212.

6. Cai HX, Liu C, Zhang JF, et al. Spontaneous epidural hematoma of thoracic spine presenting as Brown-Séquard syndrome: report of a case with review of the literature. J Spinal Cord Med. 2011;34(4):432-436.

7. Akimoto T, Yamada T, Shinoda S, et al. Spontaneous Spinal Epidural Hematoma as a Potentially Important Stroke Mimic. J Cent Nerv Syst Dis. 2014;6:15-20. 